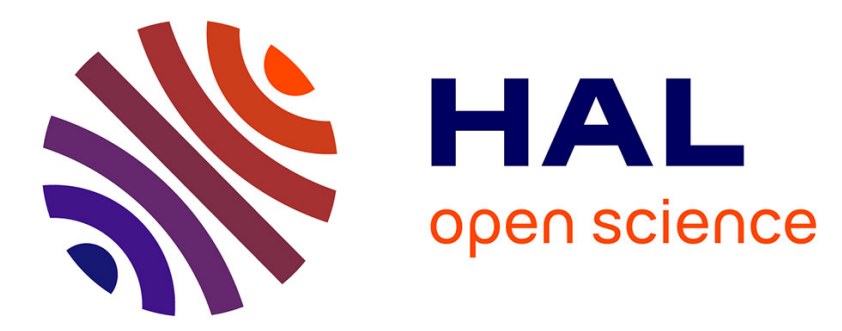

\title{
Exploration quantitative de la phonation par aérophonoscopie: étude de reproductibilité sur sujets sains
}

Laure Ganry, P. Blot, S. Balandier, J.-M. Bouric, W. Sabouni, P. Corre, R.H. Khonsari

\section{To cite this version:}

Laure Ganry, P. Blot, S. Balandier, J.-M. Bouric, W. Sabouni, et al.. Exploration quantitative de la phonation par aérophonoscopie: étude de reproductibilité sur sujets sains. Revue de Stomatologie, de Chirurgie Maxillo-faciale et de Chirurgie Orale, 2015, 117 (2), pp.62-66. 10.1016/j.revsto.2015.11.001 . hal-01253904

\section{HAL Id: hal-01253904 \\ https://hal.science/hal-01253904}

Submitted on 11 Jan 2016

HAL is a multi-disciplinary open access archive for the deposit and dissemination of scientific research documents, whether they are published or not. The documents may come from teaching and research institutions in France or abroad, or from public or private research centers.
L'archive ouverte pluridisciplinaire HAL, est destinée au dépôt et à la diffusion de documents scientifiques de niveau recherche, publiés ou non, émanant des établissements d'enseignement et de recherche français ou étrangers, des laboratoires publics ou privés. 
Exploration quantitative de la phonation par aérophonoscopie : étude de reproductibilité sur sujets sains

Quantitative study of phonation by Aerophonoscopy: reproducibility study on healthy subjects

Ganry L (1), Blot P (2), Balanger S (2), Bouric JM (2), Sabouni W (3), Corre P (4), Khonsari RH (1)

(1) AP-HP, Hôpital Pitié-Salpêtrière, Service de Chirurgie Maxillofaciale et Stomatologie, Paris, F-75013, France ; UPMC Université Paris 06, F-75005, Paris, France

(2) École d'Orthophonie, Centre Hospitalo-Universitaire de Nantes, Université de Nantes, Nantes, France

(3) Clinique d'Orthopédie Dento-Faciale La Réserve, Bandol, France

(4) Service de Chirurgie Maxillofaciale et Stomatologie, Centre Hospitalo-Universitaire de Nantes, Université de Nantes, Nantes, France

\section{Conflit d'intérêt $=$ néant}




\title{
Résumé
}

Introduction. L'Aérophonoscope permet d'enregistrer les flux d'air buccaux et nasaux lors de la respiration et de la phonation, ainsi que le son émit par le patient. Il occupe une place centrale dans la consultation de suivi des enfants présentant une fente labiopalatine en post-opératoire, mais ne dispose pas encore d'étude validant de façon quantitative sa fiabilité en situation pathologique ou non pathologique.

Matériel et Méthodes. Nous proposons une évaluation quantitative de la fiabilité de l'aérophonoscopie par un protocole de test - restest chez 30 sujets adultes sains, en mesurant sa reproductibilité inter et intra-individuelle, ainsi que sa sensibilité au degré de fermeture du sphincter vélo-pharyngé.

Résultats. L'aérophonoscope fournit des mesures quantitatives reproductibles en intra- et interindividuelle chez des sujets adultes sains. L'aérophonoscope est sensible chez le sujets sains, aux degrés d'occlusion du sphincter vélo-pharyngé.

Discussion. Une approche quantitative de l'aérophonoscopie reste à développer afin de prouver l'intérêt de ce dispositif dans le suivi des enfants présentant une fente labiopalatine et permettre de répondre à l'intérêt d'une reprise chirurgicale vélaire ou à l'efficacité de gestes vélaires secondaires comme la pharyngoplastie à pédicule inférieur ou supérieur.

\begin{abstract}
Introduction. The aerophonoscope records buccal and nasal air flow during breath and speech functions, and the sound heard from the patient. It is known as useful in the follow-up of children with cleft lip and palate after surgery, but does not have any study validating in quantitative terms its reliability in pathological or non-pathological situation.

Material and Methods. We provide an estimation of the reproducibility of the quantitative data provided by the device using a test - retest protocol in 30 healthy adult subjects, by a measure of
\end{abstract}


the inter- and intra-individual reproducibility, and its sensibility to the occlusion of the velopharyngeal sphincter,

Results. The quantitative data provided by the aerophonoscope on healthy adult subjects are reliable. The aerophonoscope is sensitive on healthy adult subjects to the occlusion of the velopharyngeal sphincter,

Conclusion. The contribution of aerophonoscopy in treatment algorithms is not well established. Reliable quantitative aerophonoscopic data would be of major interest in the evaluation of this device to follow patients with cleft lips and palate. It will lead to the answer of very controversies questions about second soft-palate operation and the real benefit of surgery procedures like pharyngoplasty with superior or inferior pedicle.

Mots-clés aérophonoscope ; fente labiopalatine ; reproductibilité de résultats ; rhinolalie ; reprise chirurgicale, suivi

Keywords aerophonoscope; cleft lip and palate; results reproducibility; rhinolalia; secondary surgery; follow-up 


\section{Introduction}

L’aérophonoscope, appareil développé par Georges Rineau en 1989, permet d'enregistrer les flux d'air buccaux et nasaux lors de la respiration et de la phonation ainsi que le son émit par le patient. L'examen aérophonoscopique permet alors d'attribuer un profil de fonctionnement vélaire aux enfants vus en consultation de suivi post-opératoire d'une reconstruction de fente palato-vélaire. En effet, l'association de l'étude des flux d'air nasaux et buccaux, ainsi que du son émit par le patient permettrait de définir objectivement une courbe ou «profil » de dysphonie. Mais aucune étude n'a pu mesurer de façon quantitative la fiabilité du dispositif, ce qui pose un problème majeur scientifique lors de la discussion thérapeutique de l'indication de reprise chirurgicale se fondant en partie sur ce profil.

D'autres dispositifs mesurant le profil vélaire existent sur le marché, mais posent des problèmes de validation de leurs résultats et/ou ou de difficultés d'utilisation en consultation lors d'un suivi à long terme.

L'aérophonoscope est un appareil nous ayant paru être un bon compromis pour permettre le suivi précis et à long terme des enfants présentant une fente labio-palatine.

Dans cette étude, nous avons procédé à une évaluation de la fiabilité des mesures quantitatives fournies par l'aérophonoscope sur une population d'adultes sains, afin de souligner l'importance d'une approche chiffrée quantitative, devant être étendue secondairement aux cas pathologiques, permettant de prouver l'intérêt diagnostique de l'aérophonoscopie chez les enfants présentant une fente labio-palatine.

La consultation de suivi de ces enfants comprend :

(1) l'examen clinique, réalisé par le chirurgien, qui contrôle l'aspect du palais, du voile et l'occlusion du sphincter vélopharyngé - la mobilisation vélaire sera contrôlée par l'émission d'un [a] tenu et, 
2) le bilan orthophonique à l'oreille (subjectif), au miroir de Glatzel (peu spécifique), et au cours duquel sont réalisés les enregistrements aérophonoscopiques.

Durant cette consultation, deux radiographies de profil sont systématiquement réalisées:

(1) au repos pour visualiser l'aspect général et la longueur du voile et,

(2) lors de l'émission d'un [i] tenu, pour en déterminer sa capacité d'élévation. 


\section{Matériel et méthodes}

L'aérophonoscope (Société Orthalis, Diedendorf, France) est composé d'une sonde comprenant deux orifices nasaux placés à la verticale des narines et un orifice buccal, d'un système de capteurs à fils chauds, un microphone externe, le tout relié à un ordinateur équipé du logiciel AÉRO RD (version 2006). Le dispositif comprend cinq canaux de saisie: (1) débit d'air nasal droit, (2) débit d'air nasal gauche, (3) débit d'air nasal total, (4) débit d'air buccal, et (5) son.

Le choix restreint de certains canaux de saisie et l'utilisation de différents temps de saisie permettent, au besoin, une analyse plus fine de la production de parole [1].

Nous avons considéré 30 volontaires sains, 17 femmes et 13 hommes, âgés de 19 à 42 ans (âge moyen: 25 ans). Aucun sujet ne présentait d'antécédent maxillo-facial, ORL ou orthophonique. Nous avons utilisé le protocole standard appliqué au CHU de Nantes (tab. 1) [1], lieu d'utilisation initiale de l'aérophonoscope. Un exemplaire écrit de ce protocole a été au préalable fourni à tous les participants, qui devaient le lire à deux reprises. Deux mesures - test et re-rest - ont été acquises. Le nombre total d'items enregistrés était 13, sur les quatre canaux (canal son exclu). L'aérophonoscope n'étant pas destiné à fournir des données quantitatives, nous avons modifié le logiciel d'acquisition avec l'assistance du constructeur. Les données brutes ainsi obtenues correspondaient à une hauteur sous la courbe exprimée sur une échelle sans unité, allant de 0 à 100. Le calibrage des quatre canaux était identique. La fréquence d'échantillonnage était de 1/125 Hz. Toutes les analyses statistiques effectuées sur les données quantitatives ont été réalisés avec le logiciel SPSS 22 (Statistical Package for the Social Sciences, IBM).

Afin d'évaluer la reproductibilité inter-individuelle, nous avons calculé la moyenne, l'écart-type, la borne inférieure et supérieure, le coefficient de variation $(\mathrm{CV})$ - qui exprime en pourcentage l'écart-type par rapport à la moyenne, avec une valeur de 100\% si l'écart-type est égal à la moyenne - et le score $\mathrm{Z}$, qui permet de positionner la valeur relevée par rapport à la moyenne et à l'écart-type de l'échantillon sur les 30 valeurs relevées du test et du re-test pour 
chaque candidat. Un score $\mathrm{Z}=0$ signifie que la valeur est égale à la moyenne; $\mathrm{Z}=1$ signifie qu'elle est égale à l'écart-type (tab. 2).

Afin de démontrer la fiabilité de l'appareil lors de mesures répétées, soit évaluer la reproductibilité intra-individuelle, nous avons calculé le coefficient de corrélation intra-classe (CCI) entre les valeurs du test et du re-test avec un modèle alpha de Cronbach, en two-way mixed, en cohérence absolue et en mesure moyenne. Une valeur CCI > 0.75 est considérée excellente en termes de fiabilité, alors qu'une valeur entre $0.45<\mathrm{CCI}<0.75$ est considérée comme correcte (tab. 3). 


\section{Résultats}

Tous les échantillons avaient une distribution normale et leur variance était homogène.

\section{Reproductibilité inter-individuelle (tab. 2)}

12 valeurs (en rouge) sur 96 (soit 12.5\% des valeurs) du CV étaient au-dessus de 70\%, 7 de ces 12 valeurs (soit 58.3\%) se trouvaient dans le groupe des voyelles orales.

\section{Reproductibilité intra-individuelle (tab. 3)}

24 valeurs du CCI sur 48 (soit 50\% des valeurs) sont au-dessus de 0.75 (bleu). Seulement 5/48 (soit 10.4\%) des valeurs sont en-dessous de 0.45 (rouge).

\section{Influence du degré de fermeture du sphincter vélo-pharyngé}

Les consonnes nasales donnent des mesures de moyennes significativement plus élevées que celles des voyelles nasales ( $p<0.05$ pour les 4 canaux, test $Z$ bilatéral), ces dernières demandant une élévation vélaire plus importante que les consonnes nasales et donc un flux nasal moins important. 


\section{Discussion}

La présente étude mesure pour la première fois des valeurs quantitatives avec l'aérophonoscope, mais nous ne disposons pas de norme chez le sujet sain pour ces valeurs.

Les 12 valeurs élevées en reproductibilité inter-individuelle indiquent que notre étude ne permet pas d'établir de telles normes. Cependant, les coefficients de variation indiquent que les valeurs de test et de re-test chez un même individu, pour un item et un canal, sont utilisables comme standard pour un sujet donné.

Notre étude indique par conséquent que l'aérophonoscope est adapté au suivi d'un patient dans le temps mais que nous n'avons pas encore la possibilité d'établir de normes caractérisant le statut de « sujet sain » pour les valeurs fournies par chaque canal.

Par ailleurs, notre étude montre que l'aérophonoscope est sensible aux degrés d'occlusion du sphincter vélo-pharyngé.

Il existe de nombreux autres dispositifs de mesure du flux d'air nasal. Le Nasomètre (Kay Elemetrics Corp., Lincoln Park) est le plus ancien dispositif de mesure quantitative de la nasalance et le plus communément utilisé. Cependant, les scores fournis par le nasomètre ne sont pas comparables d'une publication l'autre pour une situation donnée [2]. Le Nasalview (Tiger Electronics, Seattle) fournit des données non comparables à celles issues du Nasomètre [3], et l'appareil est peu adapté aux suivis à long terme [4]. L'Oronasal Mask (Glottal Enterprises, Syracuse) est encore moins fiable que les deux appareils précités en termes de reproductibilité intra-individuelle [4,5]. Au vu de ces données sur les autres dispositifs de mesure de la «nasalance », l'aérophonoscope a parfaitement sa place comme appareil de référence de quantification de la «nasalance ». Les reproductibilités inter- et intra-individuelles doivent cependant être testés chez l'enfant et dans des cas pathologiques avant de conclure définitivement sur la fiabilité de ce dispositif.

Une fois que la fiabilité de l'aérophonoscope sera démontrée dans les cas pathologiques, cet appareil pourra être utilisé pour évaluer quantitativement les effets de la chirurgie secondaire 
des fentes vélaires, dont les résultats n'ont pas encore fait l'objet de larges études chiffrées. Ce dispositif pourrait intervenir aux moments clés du schéma thérapeutique, notamment lors de la décision de pratiquer une chirurgie secondaire à visée phonatoire. Les données qualitatives actuellement données par la machine dans les cas pathologiques (fig. 1-3) sont indicatives en pratique clinique mais ne permettent pas d'établir des conduites à tenir rigoureuses. Des données quantitatives seraient également d'un intérêt majeur pour confirmer les effets bénéfiques de gestes chirurgicaux controversés, tels que la pharyngoplastie à pédicule inférieur ou supérieur [6]. 
1. Blot P, Khonsari RH. Mesure du flux nasal et aérophonoscopie. Rev Stomatol Chir Maxillofac 2009;110:89-93

2. Mayo R, Floyd L, Warren D, Dalston R, Mayo C. Nasalance and nasal area values: crossracial study. Cleft palate craniofac J 1996; 33: 143-149

3. Lewis K, Watterson T. Comparison of Nasalance scores obtained from the Nasometer and the Nasalview. Cleft palate craniofac J 2003; 40: 40-45

4. Bressmann T. Comparison of nasalance scores obtained with the Nasometer, the Nasalview and the OroNasal System. Cleft palate craniofac J 2005; 42: 423-433

5. Bressmann T, Klaiman P, Fischbach S. Same noses, different nasalance scores: Data from normal subjects and cleft palate speakers for three systems for nasalance analysis. Clin Linguist Phon 2006 20:163-170

6. Gbaguidi C., Testelin S., Devauchelle B. Les pharyngoplasties dans le traitement de l'insuffisance vélo-pharyngée des fentes palatines. Rééducation orthophonique $2003 ; 216: 65-77$ 


\section{Légendes des tableaux}

Tableau 1. Protocole d'acquisition aérophonoscopique.

Table 1. Aerophonoscopy : acquisition protocol.

Tableau 2. Reproducibilité inter-individuelle des mesures de l'aérophonoscope. S1 : test ; S2 : retest .

Table 2. Inter-subject reproducibility of the quantitative values provided by the aerophonoscope. S1 : test ; S2 : retest .

Tableau 3. Reproducibilité intra-individuelle des mesures de l'aérophonoscope. S1 : test ; S2 : retest ; CCI : coefficient de corrélation intra-classe. En bleu : CCI < 0.75 (excellent); en rouge : CCI $<0.45$ (mauvais).

Table 3. Intra-subject reproducibility of the quantitative values provided by the aerophonoscope. $\mathrm{S} 1$ : test; $\mathrm{S} 2$ : retest ; CCI : intra-class correlation. In blue : CCI > 0.75 (excellent); in red : CCI $<0.45$ (poor) 


\section{Légendes des figures}

Figure 1. Rhinolalie ouverte fonctionnelle: déperditions nasales plus importantes pour les voyelles fermées que pour les voyelles ouvertes

Figure 2. Rhinolalie ouverte fonctionnelle: déperditions nasales plus importantes pour les consonnes fricatives que pour les consonnes occlusives.

Figure 3. Rhinolalie ouverte fonctionnelle: déperditions nasale moins importantes pour les phrases standard (1) que pour les phrases en [i] (2). 


\section{$\underline{\text { Tableaux }}$}


1. Perméabilité nasale - émission des mots 'NON MAMAN, NON MAMAN' + respiration de repos

2. Mobilisation passive / tonicité du voile - émission d'un souffle buccal tenu

3. Mobilisation active du voile 1/3 - émission de voyelles orales isolées ouvertes puis fermées

4. Mobilisation active du voile $2 / 3$ - émission de syllabes isolées consonnes occlusives sourdes [PA TA KA] consonnes occlusives sonores [BA DA GA] consonnes fricatives [FA SA CHA]

5. Mobilisation active du voile $\mathbf{3 / 3}$-émission de phrases orales 
Tableau 1

\begin{tabular}{|c|c|c|c|c|}
\hline Paramètres & Site & Moyenne & Écart type & $C V(\%)$ \\
\hline \multirow[t]{8}{*}{ Perméabilité nasale } & Nasal R S1 & 80,41 & 20,75 & 25,8 \\
\hline & Nasal R S2 & 76,44 & 23,90 & 31,26 \\
\hline & Nasal L S1 & 88,72 & 9,35 & 10,54 \\
\hline & Nasal L S2 & 82,27 & 19,43 & 23,62 \\
\hline & Nasal Total S1 & 85,69 & 11,12 & 12,98 \\
\hline & Nasal Total S2 & 80,38 & 15,19 & 18,89 \\
\hline & Oral S1 & 17,62 & 12,94 & 73,41 \\
\hline & Oral S2 & 14,51 & 8,59 & 59,20 \\
\hline \multirow[t]{8}{*}{ Voyelles orales } & Nasal R S1 & 11,82 & 13,77 & 116,48 \\
\hline & Nasal R S2 & 8,59 & 4,46 & 51,97 \\
\hline & Nasal L S1 & 8,86 & 16,76 & 189,22 \\
\hline & Nasal L S2 & 5,17 & 7,27 & 140,52 \\
\hline & Nasal Total S1 & 10,11 & 14,99 & 148,17 \\
\hline & Nasal Total S2 & 5,62 & 4,36 & 77,60 \\
\hline & Oral S1 & 10,51 & 10,11 & 96,23 \\
\hline & Oral S2 & 11,31 & 9,33 & 82,51 \\
\hline \multirow[t]{7}{*}{ Voyelles nasales } & Nasal R S1 & 63,18 & 26,61 & 42,12 \\
\hline & Nasal R S2 & 59,82 & 27,44 & 45,87 \\
\hline & Nasal L S1 & 74,18 & 24,71 & 33,32 \\
\hline & Nasal L S2 & 67,54 & 24,48 & 36,25 \\
\hline & Nasal Total S1 & 69,88 & 20,31 & 29,06 \\
\hline & Nasal Total S2 & 64,16 & 18,66 & 29,09 \\
\hline & Oral S1 & 7,10 & 6,27 & 88,24 \\
\hline
\end{tabular}




\begin{tabular}{|c|c|c|c|c|}
\hline & Oral S2 & 9,43 & 11,72 & 124,23 \\
\hline \multirow[t]{8}{*}{ Consonnes nasales } & Nasal R S1 & 81,42 & 23,66 & 29,06 \\
\hline & Nasal R S2 & 74,34 & 27,63 & 37,17 \\
\hline & Nasal L S1 & 81,79 & 26,24 & 32,08 \\
\hline & Nasal L S2 & 79,87 & 26,50 & 33,17 \\
\hline & Nasal Total S1 & 86,37 & 15,10 & 17,49 \\
\hline & Nasal Total S2 & 82,38 & 14,30 & 17,36 \\
\hline & Oral S1 & 4,12 & 2,81 & 68,30 \\
\hline & Oral S2 & 4,12 & 2,08 & 50,49 \\
\hline \multirow[t]{8}{*}{ Occlusives sourdes + voyelles ouvertes } & Nasal R S1 & 15,75 & 3,31 & 20,99 \\
\hline & Nasal R S2 & 13,40 & 4,13 & 30,86 \\
\hline & Nasal L S1 & 9,39 & 4,72 & 50,27 \\
\hline & Nasal L S2 & 7,78 & 5,13 & 65,94 \\
\hline & Nasal Total S1 & 12,05 & 3,41 & 28,29 \\
\hline & Nasal Total S2 & 10,09 & 3,56 & 35,35 \\
\hline & Oral S1 & 19,85 & 9,25 & 46,61 \\
\hline & Oral S2 & 17,21 & 7,58 & 44,04 \\
\hline \multirow[t]{8}{*}{ Occlusives sourdes + voyelles fermées } & Nasal R S1 & 15,26 & 3,02 & 19,77 \\
\hline & Nasal R S2 & 13,12 & 3,35 & 25,50 \\
\hline & Nasal L S1 & 8,27 & 4,77 & 57,63 \\
\hline & Nasal L S2 & 7,14 & 3,75 & 52,50 \\
\hline & Nasal Total S1 & 11,33 & 3,17 & 27,96 \\
\hline & Nasal Total S2 & 9,64 & 1,92 & 19,90 \\
\hline & Oral S1 & 21,36 & 8,20 & 38,40 \\
\hline & Oral S2 & 19,69 & 7,10 & 36,08 \\
\hline
\end{tabular}




\begin{tabular}{|c|c|c|c|c|}
\hline \multirow[t]{8}{*}{ Occlusives sonores + voyelles ouvertes } & Nasal R S1 & 14,74 & 3,68 & 24,97 \\
\hline & Nasal R S2 & 13,22 & 3,56 & 26,90 \\
\hline & Nasal L S1 & 9,97 & 6,63 & 66,48 \\
\hline & Nasal L S2 & 7,47 & 4,35 & 58,15 \\
\hline & Nasal Total S1 & 11,87 & 5,01 & 42,19 \\
\hline & Nasal Total S2 & 9,99 & 3,17 & 31,73 \\
\hline & Oral S1 & 14,50 & 7,20 & 49,65 \\
\hline & Oral S2 & 12,41 & 5,88 & 47,41 \\
\hline \multirow[t]{8}{*}{ Occlusives sonores + voyelles fermées } & Nasal R S1 & 15,19 & 4,24 & 27,93 \\
\hline & Nasal R S2 & 13,80 & 3,76 & 27,25 \\
\hline & Nasal L S1 & 9,20 & 5,36 & 58,29 \\
\hline & Nasal L S2 & 7,45 & 3,87 & 51,97 \\
\hline & Nasal Total S1 & 12,08 & 4,09 & 33,86 \\
\hline & Nasal Total S2 & 10,18 & 2,91 & 28,62 \\
\hline & Oral S1 & 18,46 & 8,91 & 48,28 \\
\hline & Oral S2 & 16,60 & 8,86 & 53,41 \\
\hline \multirow[t]{8}{*}{ Fricatives + voyelles ouvertes } & Nasal R S1 & 15,76 & 8,99 & 57,08 \\
\hline & Nasal R S2 & 14,13 & 4,72 & 33,42 \\
\hline & Nasal L S1 & 9,61 & 6,05 & 62,99 \\
\hline & Nasal L S2 & 9,44 & 9,33 & 98,88 \\
\hline & Nasal Total S1 & 12,08 & 7,21 & 59,71 \\
\hline & Nasal Total S2 & 11,04 & 5,79 & 52,45 \\
\hline & Oral S1 & 39,20 & 16,98 & 43,32 \\
\hline & Oral S2 & 37,22 & 19,21 & 51,61 \\
\hline Fricatives + voyelles fermées & Nasal R S1 & 14,64 & 4,25 & 28,99 \\
\hline
\end{tabular}




\begin{tabular}{|c|c|c|c|c|}
\hline \multicolumn{2}{|r|}{ Nasal R S2 } & 12,86 & 3,92 & 30,49 \\
\hline & Nasal L S1 & 8,70 & 3,82 & 43,88 \\
\hline & Nasal L S2 & 8,55 & 7,49 & 87,65 \\
\hline & Nasal Total S1 & 11,33 & 2,81 & 24,78 \\
\hline & Nasal Total S2 & 10,89 & 4,91 & 45,10 \\
\hline & Oral S1 & 43,18 & 16,94 & 39,22 \\
\hline & Oral S2 & 40,71 & 17,10 & 42,01 \\
\hline \multirow[t]{8}{*}{ Phrases orales } & Nasal R S1 & 16,22 & 3,17 & 19,56 \\
\hline & Nasal R S2 & 15,31 & 3,82 & 24,94 \\
\hline & Nasal L S1 & 8,82 & 3,98 & 45,09 \\
\hline & Nasal L S2 & 8,63 & 4,07 & 47,19 \\
\hline & Nasal Total S1 & 11,83 & 2,61 & 22,02 \\
\hline & Nasal Total S2 & 11,83 & 2,89 & 24,39 \\
\hline & Oral S1 & 34,77 & 14,06 & 40,43 \\
\hline & Oral S2 & 33,13 & 12,04 & 36,36 \\
\hline \multirow[t]{8}{*}{ Phrases nasales } & Nasal R S1 & 13,11 & 4,85 & 37,01 \\
\hline & Nasal R S2 & 14,61 & 4,79 & 32,79 \\
\hline & Nasal L S1 & 8,61 & 5,69 & 66,10 \\
\hline & Nasal L S2 & 8,81 & 5,55 & 63,00 \\
\hline & Nasal Total S1 & 10,31 & 4,34 & 42,07 \\
\hline & Nasal Total S2 & 11,06 & 4,69 & 42,35 \\
\hline & Oral S1 & 94,20 & 2,24 & 2,38 \\
\hline & Oral S2 & 94,86 & 0,93 & 0,98 \\
\hline
\end{tabular}




\section{Tableau 2.}

\begin{tabular}{|c|c|c|}
\hline Paramètre & Site & CCI \\
\hline \multirow[t]{4}{*}{ Perméabilité nasale } & S1/S2 nasal droit & 0,73 \\
\hline & S1/S2 nasal gauche & 0,59 \\
\hline & S1/S2 nasal total & 0,79 \\
\hline & $\mathrm{S} 1 / \mathrm{S} 2$ oral & 0,67 \\
\hline \multirow[t]{4}{*}{ Voyelles orales } & S1/S2 nasal droit & 0,37 \\
\hline & S1/S2 nasal gauche & 0,77 \\
\hline & $\mathrm{S} 1 / \mathrm{S} 2$ nasal total & 0,44 \\
\hline & $\mathrm{S} 1 / \mathrm{S} 2$ oral & 0,77 \\
\hline \multirow[t]{4}{*}{ Voyelles nasales } & S1/S2 nasal droit & 0,81 \\
\hline & S1/S2 nasal gauche & 0,81 \\
\hline & S1/S2 nasal total & 0,79 \\
\hline & $\mathrm{S} 1 / \mathrm{S} 2$ oral & 0,49 \\
\hline \multirow[t]{4}{*}{ Consonnes nasales } & S1/S2 nasal droit & 0,81 \\
\hline & S1/S2 nasal gauche & 0,73 \\
\hline & S1/S2 nasal total & 0,67 \\
\hline & $\mathrm{S} 1 / \mathrm{S} 2$ oral & 0,72 \\
\hline \multirow[t]{2}{*}{ Occlusives sourdes + voyelles ouvertes } & $\mathrm{S} 1 / \mathrm{S} 2$ nasal droit & 0,56 \\
\hline & S1/S2 nasal gauche & 0,39 \\
\hline
\end{tabular}




\begin{tabular}{|c|c|c|}
\hline & S1/S2 nasal total & 0,37 \\
\hline & S1/S2 oral & 0,77 \\
\hline Occlusives sourdes + voyelles fermées & S1/S2 nasal droit & 0,65 \\
\hline & S1/S2 nasal gauche & 0,78 \\
\hline & S1/S2 nasal total & 0,57 \\
\hline & $\mathrm{S} 1 / \mathrm{S} 2$ oral & 0,76 \\
\hline Occlusives sonores + voyelles ouvertes & S1/S2 nasal droit & 0,77 \\
\hline & S1/S2 nasal gauche & 0,71 \\
\hline & S1/S2 nasal total & 0,77 \\
\hline & $\mathrm{S} 1 / \mathrm{S} 2$ oral & 0,87 \\
\hline Occlusives sonores + voyelles fermées & S1/S2 nasal droit & 0,78 \\
\hline & S1/S2 nasal gauche & 0,63 \\
\hline & S1/S2 nasal total & 0,60 \\
\hline & $\mathrm{S} 1 / \mathrm{S} 2$ oral & 0,77 \\
\hline Fricatives + voyelles ouvertes & S1/S2 nasal droit & 0,56 \\
\hline & S1/S2 nasal gauche & 0,87 \\
\hline & S1/S2 nasal total & 0,93 \\
\hline & $\mathrm{S} 1 / \mathrm{S} 2$ oral & 0,87 \\
\hline Fricatives + voyelles fermées & S1/S2 nasal droit & 0,81 \\
\hline & S1/S2 nasal gauche & 0,47 \\
\hline & S1/S2 nasal total & 0,59 \\
\hline
\end{tabular}




\begin{tabular}{|l|l|c|} 
& S1/S2 oral & 0,88 \\
\hline Phrases orales & S1/S2 nasal droit & 0,84 \\
\cline { 2 - 3 } & S1/S2 nasal gauche & 0,76 \\
\cline { 2 - 3 } & S1/S2 nasal total & 0,84 \\
\cline { 2 - 3 } Phrases nasales & S1/S2 oral & 0,90 \\
\hline & S1/S2 nasal droit & 0,57 \\
\hline & S1/S2 nasal gauche & 0,56 \\
\hline & S1/S2 nasal total & 0,60 \\
\hline & S1/S2 oral & 0,09 \\
\hline & & \\
\hline
\end{tabular}




\section{Figures}

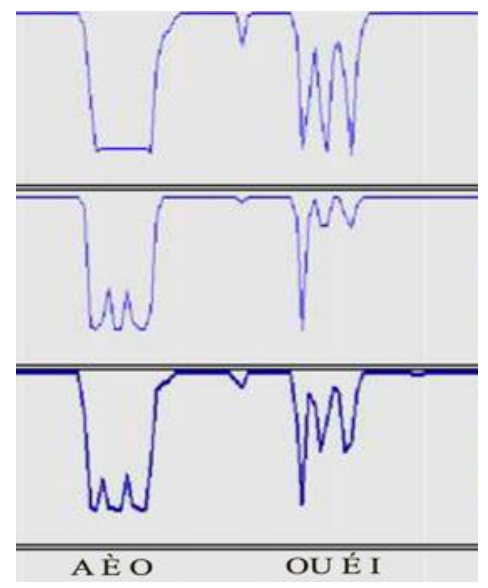

Figure 1

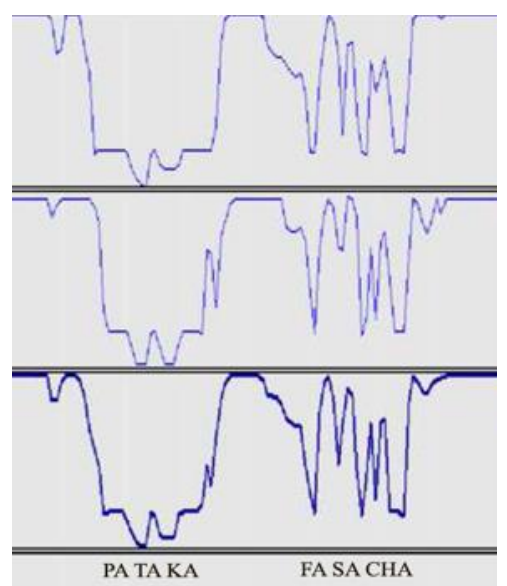

Figure 2

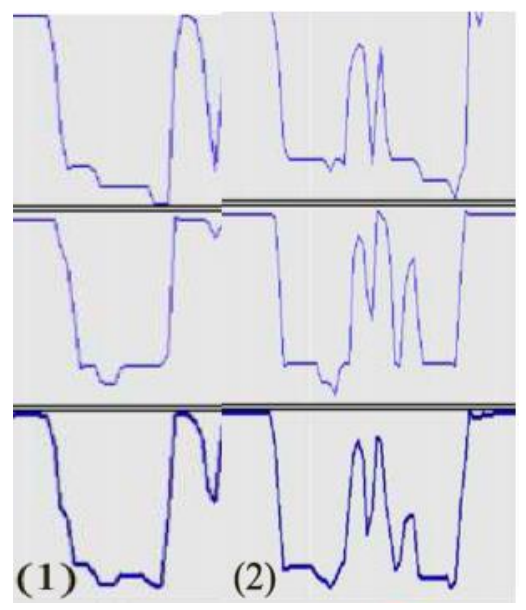

Figure 3 